\title{
Does testosterone affect emotional perception after social approach-avoidance?
}

Sina Radke*, Inge Volman, Pranjal Mehta, Veerle van Son, Dorien Enter, Alan Sanfey, Ivan

Toni, Ellen R. A. de Bruijn, Karin Roelofs

This work was conducted at the Radboud University Nijmegen, Donders Institute for Brain, Cognition and Behaviour, Nijmegen, The Netherlands, and presents supplementary results to the following publication:

S. Radke, I. Volman, P.H. Mehta, V. van Son, D. Enter, A.G. Sanfey, I. Toni, E.R.A. De Bruijn, K. Roelofs, Testosterone biases the amygdala toward social threat approach. Science Advances 1, e1400074 (2015). DOI: 10.1126/sciadv.1400074

*Corresponding author at RWTH Aachen, Faculty of Medicine, Department of Psychiatry, Psychotherapy and Psychosomatics, Pauwelsstr. 30, 52074 Aachen, Germany; sradke@ukaachen.de

Keywords: testosterone, amygdala, social approach, approach-avoidance, motivational salience

\section{Introduction}

Testosterone may promote approach-related behaviors by modulating sensitivity to social threat (for a review see 1). In humans, testosterone increases amygdala activation to angry facial expressions (2-4), specifically when threat approach is required (5). As both increased and decreased threat detection after testosterone administration have been reported (6-9), we aim to contribute to the discussion by sharing additional results, i.e., perception ratings, from our previously published findings (5). Here, a single dose of $0.5 \mathrm{mg}$ of testosterone increased amygdala activation during threat approach, and decreased it during threat avoidance. After the MRI session, about 5 hours post-administration, participants rated their emotional perception of the faces to which they had made approach and avoidance actions in the social approachavoidance task.

\section{Materials and Methods}

Out of the 54 female participants performing the social approach-avoidance task (5), 53 subsequently rated the stimuli ( $n=26$ testosterone; $n=27$ placebo). In the course of the original study, written informed consent was obtained from all participants, with the protocol being approved by the CMO Nijmegen-Arnhem in accordance with the Declaration of Helsinki. 
The rating was conducted after the MRI session, outside the MR scanner on a PC, about 5 minutes after the last saliva sample was obtained, i.e., 5 hours post-administration, when testosterone levels were still elevated (compare Table 1 in (5)).

Participants rated the affective expression of all stimuli used in the social approach-avoidance task (36 angry, 36 happy faces). In randomized order, stimuli were presented in the center of the screen until a response was given. Participants were instructed to base their rating on a bipolar scale with 1 = "very angry" and 7 = "very happy" as verbal endpoints (and 4 = "neutral"). Presentation of stimuli and acquisition of responses were controlled by Presentation software version 13.

Mean ratings were calculated for each emotion (angry, happy). Normal distribution was verified via Kolmogorov-Smirnov tests before the rating data were subjected to a repeated measures ANOVA, with the within-subject factor Emotion (angry, happy) and the between-subjects factor Substance (testosterone, placebo). As in the RT analyses of the social approach-avoidance task (5), the standardized testosterone and cortisol levels from the first saliva measurement were included in the ANOVA as covariates. Within-subject effects with Greenhouse Geisser correction are reported.

In addition, we tested whether the rating of angry faces was related to amygdala activation. As amygdala activation significantly differed between approach and avoidance after testosterone administration (5), this difference score (approach angry - avoid angry) was correlated (Pearson's correlation coefficient) with the mean rating for angry faces per Substance group. For all analyses, the a-level was set at $P<0.05$. Statistical testing was performed with the Statistical Package for the Social Sciences (IBM SPSS 25).

\section{Results}

There was a main effect of Emotion $\left(F_{1,49}=2266.64, P<0.001\right.$, partial $\left.\eta^{2}=0.98\right)$, due to higher ratings for happy $(M=5.89 ; S D=0.35)$ than for angry faces $(M=2.23, S D=0.35)$.

The Emotion $x$ Substance interaction showed a trend toward significance $\left(F_{1,49}=3.0, P=0.089\right.$, partial $\left.\eta^{2}=0.06\right)$, but all other effects, i.e., of Substance or involving the covariates were not (nearly) significant (all Fs $<0.8, P s>0.38$ ).

Decomposing the Emotion $x$ Substance interaction revealed a marginally significant Substance effect for angry faces $\left(F_{1,49}=3.46, P=0.069\right.$, partial $\left.\eta^{2}=0.07\right)$, but not for happy faces $\left(F_{1,49}=\right.$ $0.90, P=0.35$, partial $\eta^{2}=0.02$ ). As the means in Table 1 indicate, participants in the testosterone group tended to rate the angry faces as more angry than participants in the placebo group.

Ratings for angry faces were not correlated with amygdala activation $(r=-0.13, P=0.53$ for placebo; $r=-0.17, P=0.40$ for testosterone, respectively). 
Table1. Means (SD) of the stimulus rating (scale from 1 to 7 ). $P$-values indicate differences between substance groups.

\begin{tabular}{|l|l|l|l|}
\hline & Placebo $(\mathrm{n}=26)$ & Testosterone $(\mathrm{n}=27)$ & $P$-value \\
\hline Angry faces & $2.31(0.30)$ & $2.14(0.39)$ & 0.069 \\
\hline Happy faces & $5.84(0.39)$ & $5.93(0.29)$ & 0.35 \\
\hline
\end{tabular}

\section{References}

1. S.N. Geniole, J.M. Carré, Human social neuroendocrinology: Review of the rapid effects of testosterone. Horm. Behav. (2018). doi: 10.1016/j.yhbeh.2018.06.001

2. S.M.M. Goetz, L. Tang, M.E. Thomason, M.P. Diamond, A.R. Hariri, J.M. Carré, Testosterone rapidly increases neural reactivity to threat in healthy men: A novel two-step pharmacological challenge paradigm. Biol. Psychiatry 76, 324-331 (2014).

3. E.J. Hermans, N.F. Ramsey, J. van Honk, Exogenous testosterone enhances responsiveness to social threat in the neural circuitry of social aggression in humans. Biol. Psychiatry 63, 263-270 (2008).

4. G.A. van Wingen, S.A. Zylicz, S. Pieters, C. Mattern, R.J. Verkes, J.K. Buitelaar, G. Fernandez, Testosterone increases amygdala reactivity in middle-aged women to a young adulthood level. Neuropsychopharmacology 34, 539-547 (2009).

5. S. Radke, I. Volman, P.H. Mehta, V. van Son, D. Enter, A.G. Sanfey, I. Toni, E.R.A. De Bruijn, K. Roelofs, Testosterone biases the amygdala toward social threat approach. Science Advances 1, e1400074 (2015).

6. D. Terburg, H. Aarts, J. van Honk, Testosterone affects gaze aversion from angry faces outside of conscious awareness. Psychol. Sci. 23, 459-463 (2012).

7. J. van Honk, D.J.L.G. Schutter, Testosterone reduces conscious detection of signals serving social correction - implications for antisocial behavior. Psychol. Sci. 18, 663-667 (2007).

8. J. van Honk, A. Tuiten, M. van den Hout, H. Koppeschaar, J. Thijssen, E. de Haan, R. Verbaten, Conscious and preconscious selective attention to social threat: Different neuroendocrine response patterns. Psychoneuroendocrinology 25, 577-591 (2000).

9. J.M. van Peer, D. Enter, H. van Steenbergen, P. Spinhoven, K. Roelofs, Exogenous testosterone affects early threat processing in socially anxious and healthy women. Biol. Psychol. 129, $82-89$ (2017). 